\title{
A CHEMOTYPING SCHEME FOR CLINICAL ISOLATES \\ OF HAEMOPHILUS INFLUENZAE
}

\author{
G. M. TebButt
}

\section{Public Health Laboratory, South Cleveland Hospital, Marton Road, Middlesbrough, TS4 $3 T$ A}

\begin{abstract}
Summary. A total of 464 Haemophilus influenzae strains, most of them fresh clinical isolates, have been classified by chemotyping-a combination of auxotyping and biotyping. Seven auxotests and four other biochemical tests allowed recognition of 56 types. These were to a degree site-specific. $H$. influenzae of capsular type b proved almost without exception to belong to one chemotype, and 24 of 33 strains assigned to this chemotype were capsulated. When surgical-ward isolates of $H$. influenzae were typed, the results suggested that some cross-infection had occurred.
\end{abstract}

\section{INTRODUCTION}

Although uncommon, secondary cases of disease due to Haemophilus influenzae of capsular type b are well documented, and can occur within a patient's family or among close non-family contacts (Glode et al., 1980). Their secondary nature is recognisable because of the micro-organism's possession of the type $b$ capsule. Dissemination of non-capsulated strains of $H$. influenzae undoubtedly also occurs, but the frequency of transmission and the importance of hospital-acquired and community-acquired infections with these are unknown. Outbreaks of infection with non-capsulated strains of $H$. influenzae, recognisable because they produced $\beta$-lactamase, have been described (Malmvall and Branefors-Helander, 1978; Mehtar and Law, 1980). Other outbreaks may have passed unnoticed for lack of any such conspicuous strain-marker. Biotyping, based on the ability of an isolate to produce indole, urease and ornithine decarboxylase (Kilian, 1976), provides some information, but does not by itself provide an epidemiologically useful level of strain discrimination. Use of antibiograms (Ellis et al., 1978) has similar limitations. Although isolates of $H$. influenzae type $b$ have been subtyped successfully by the analysis of their outer-membrane proteins, studies with non-capsulated strains by means of this technique have been less successful (Barenkamp, Munson and Granoff, 1982).

Several workers have investigated the growth of $H$. influenzae in chemically defined media for genetic or metabolic studies (Butler, 1962; Wolin, 1963; Herriott et al., 1970). The results suggest that strains differ in their nutritional requirements. However, only a limited number of highly selected strains were examined by these workers. The 
present study was undertaken to investigate further the nutritional heterogeneity shown by strains of $H$. influenzae, and to see whether, in combination with biotyping, it could be the basis for a reliable typing scheme for clinical isolates of $H$. influenzae.

\section{MATERIALS AND METHODS}

\section{Strains of $H$. influenzae}

Except for two type strains of $H$. influenzae, NCTC nos. 4560 (non-capsulated) and 8467 (capsular type b), the strains were routine clinical isolates; 309 were from sputum, 83 from eyes, 43 from noses and throats, 27 from ears or antral wash-outs, 6 from female genital tracts, and 8 from blood or cerebrospinal fluid.

All strains showed satellite growth on Columbia Agar (Oxoid CM331) around a paper disk that contained haemin and nicotinamide adenine dinucleotide (NAD); they were non-haemolytic on horse-blood agar and were unable to synthesise porphyrins from $\delta$-aminolaevulinic acid (Kilian, 1974). All strains that produced mucoid colonies on the defined medium (see below), and some that produced non-mucoid colonies, were examined for capsules by means of a coagglutination test kit (Phadebact Haemophilus Test) obtained from Pharmacia (Great Britain) Ltd, Hounslow, Middlesex; this contains one reagent to identify type $b$ strains and another to detect (but not differentiate between) capsulated strains of the other five types (a, c-f).

All isolates were suspended in nutrient broth (Oxoid No. 2) with glycerol $10 \%(\mathrm{v} / \mathrm{v})$, and were either snap frozen and stored in liquid nitrogen, or frozen at $-70^{\circ} \mathrm{C}$.

\section{Media}

Isolation medium. Strains were isolated on Columbia Agar containing horse blood (Gibco) $5 \%(\mathrm{v} / \mathrm{v}), \mathrm{NAD} 10 \mathrm{mg} / \mathrm{L}$, and haemin $10 \mathrm{mg} / \mathrm{L}$. Bacitracin $300 \mathrm{mg} / \mathrm{L}$ was added to the medium used for some throat swabs.

Defined medium. Initial studies were performed with various defined media (table I), which were progressively modified as described in the text. In some experiments the following substances (quantities expressed as $\mathrm{mg} / \mathrm{L}$ of complete medium) were used: L-ornithine (100), L-threonine (100), L-proline (100), thymidine (100), riboflavin (1), choline chloride (1), and spermine hydrochloride (50). The media were solidified with agar (Oxoid No. $11 \%$, or Difco Bacto-Agar $1.2 \%$, or New Zealand (Davis) $1 \%$ ), or with agarose (BDH) $1 \%$. The composition of the final medium (designated GMT) is shown in table I. To study the nutritional requirements of different strains of $H$. influenzae, a set of typing media was prepared by omitting the appropriate factor or factors from the complete medium.

Inoculation of the defined medium. Bacteria were harvested into quarter-strength Ringer solution (Oxoid), and large clumps of bacteria were broken up by agitating the suspension for about $10 \mathrm{~s}$ on a vortex mixer. Each suspension was adjusted so that it contained approximately $10^{8}$ bacteria $/ \mathrm{ml}$ (barium sulphate standard no. 1), and diluted tenfold in Ringer solution. A Denley multipoint inoculator (Denley Instruments Ltd, Billingshurst, Sussex) was used to inoculate the various media with c. $2 \times 10^{4} \mathrm{cfu}$ of each micro-organism. A surface viable count was performed on several strains to check the inoculum size. Cultures were incubated in $\mathrm{CO}_{2}$ $5 \%$ in air for $24 \mathrm{~h}$ at $37^{\circ} \mathrm{C}$. No growth or considerably diminished growth (minute colonies or a slight haze) was recorded as a negative result.

\section{Biochemical tests}

Test reagents were stored in 5-ml amounts at $-20^{\circ} \mathrm{C}$. For use, a 5-ml sample was thawed and kept for up to 7 days at $4^{\circ} \mathrm{C}$. Indole tests were performed in glass tubes (Dreyer's tubes), and microtitre trays were used for other tests. For each isolate, a heavy bacterial suspension was prepared in saline, and $25 \mu \mathrm{l}$ of the suspension was mixed with $50 \mu \mathrm{l}$ of test reagent.

Indole production. This was tested as described by Clarke and Cowan (1952). Tests were incubated for $2 \mathrm{~h}$ at $37^{\circ} \mathrm{C}$ before addition of Kovács' reagent.

Urease activity. The method described by Lautrop (1960) was used. After inoculation, 
several drops of sterile paraffin oil were added to each test. The results were read after incubation for $4 \mathrm{~h}$ at $37^{\circ} \mathrm{C}$.

Ornithine or lysine decarboxylase activity. The medium was similar to that described by Møller (1955). After inoculation, several drops of sterile paraffin oil were added to each test. A control without aminoacid was included for each isolate tested. The results were read after incubation for $4 \mathrm{~h}$ at $37^{\circ} \mathrm{C}$ and then after overnight at room temperature.

\section{RESULTS}

\section{Typing medium}

Preliminary experiments showed that none of the three previously described defined media solidified with agar (for details see table I) was adequate for growth of a wide range of strains. To some degree, bacteriological-grade agars inhibited growth of $H$. influenzae and this inhibition was not eliminated by adding sodium oleate to the medium. When agarose was added in place of agar, all except two of 60 strains grew on Herriott's medium, but they grew less well on the other defined media. The addition of glucose and thiamine pyrophosphate to Herriott's medium increased the colony size, and occasional strains grew better when oxaloacetate, acetate, and ferric nitrate were added. Inosine did not improve the growth of any of the strains, and was omitted from the complete medium. Thymidine was also included in the complete medium, but was not essential for the growth of any of the 60 strains tested. With these exceptions, the composition of the final medium GMT was similar to that described by Herriott et al. (1970).

Further preliminary tests showed that arginine, haemin, NAD, pantothenate, cysteine and uracil were essential for the growth of all 60 strains on the defined medium. Thiamine pyrophosphate, glutamic acid and aspartic acid were needed for optimal growth. Whereas cystine could replace cysteine, ornithine did not eliminate a requirement for arginine in the medium. Histidine or hypoxanthine or both were essential for the growth of some strains, suggesting that these factors could be useful in typing studies. Although certain aminoacids, notably serine, stimulated the growth of some strains of $H$. influenzae on the medium, an absolute requirement for an aminoacid, other than arginine, cysteine, histidine, glutamic acid and aspartic acid, was uncommon ( 2 out of 60 strains tested). For typing studies, three variants of GMT medium were selected: (1) a medium omitting histidine, (2) a medium omitting hypoxanthine, and (3) a medium omitting glycine, isoleucine, leucine, lysine, methionine, serine, thymidine, tryptophan and tyrosine (designated aminoacid-thymidine-depleted). Isolates that grew on the complete GMT medium but not on the aminoacid-thymidine-depleted medium were retested on media prepared by omitting one factor at a time from the complete medium.

During the study, 478 strains of $H$. influenzae were tested on GMT medium. Of these, $14(2.9 \%)$ failed to grow, and a further 13 strains $(2.7 \%)$ required subculture on the medium before satisfactory growth was obtained for typing studies. Four of the 14 strains that failed to grow on the complete medium grew well on the aminoacid-thymidine-depleted medium. Further studies showed that thymidine inhibited the growth of these strains. Twenty-seven strains produced large mucoid colonies on GMT medium, and coagglutination tests confirmed that these micro-organisms were capsulated. None of 25 strains that produced non-mucoid colonies gave a positive 
TABLE I

Composition of defined media for $H$. influenzae

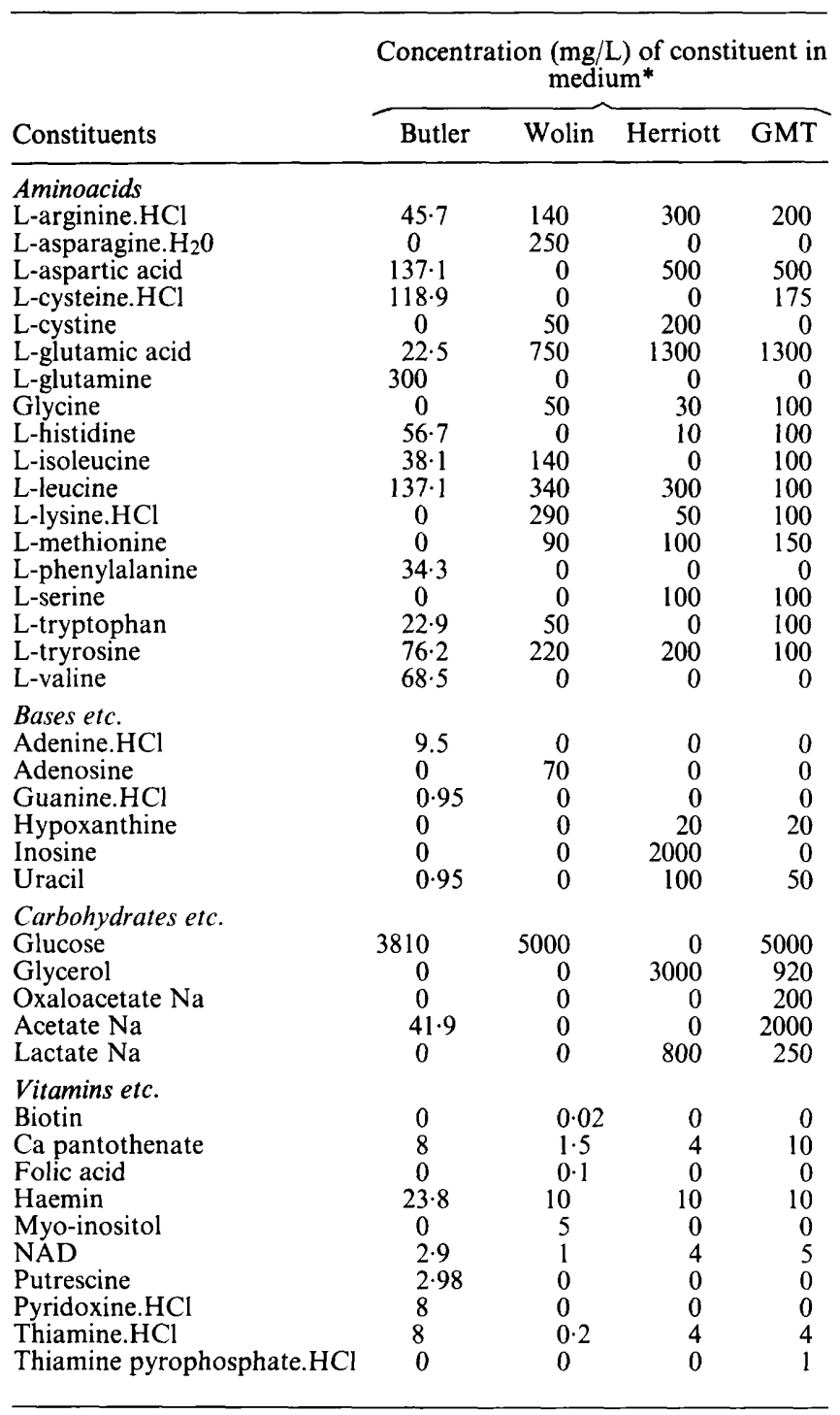


TABLE 1 (cont.)

\begin{tabular}{|c|c|c|c|c|}
\hline \multirow[b]{2}{*}{ Constituents } & \multicolumn{4}{|c|}{$\begin{array}{c}\text { Concentration }(\mathrm{mg} / \mathrm{L}) \text { of constituent in } \\
\text { medium }\end{array}$} \\
\hline & Butler & Wolin & Herriott & GMT \\
\hline \multicolumn{5}{|l|}{ Inorganic salts } \\
\hline $\mathrm{CaCl}_{2}$ & & 10 & 22 & 37 \\
\hline $\mathrm{Fe}\left(\mathrm{NO}_{3}\right)_{3} .9 \mathrm{H}_{2} \mathrm{O}$ & 0 & 0 & 0 & 2 \\
\hline $\mathrm{FeSO}_{4} .7 \mathrm{H}_{2} \mathrm{O}$ & $1 \cdot 14$ & 0 & 0 & 0 \\
\hline $\mathrm{K}_{2} \mathrm{HPO}_{4}$ & 3300 & 670 & 3500 & 3480 \\
\hline $\mathrm{K}_{2} \mathrm{SO}_{4}$ & 0 & 0 & 1000 & 1000 \\
\hline $\mathrm{MgCl}_{2} \cdot 6 \mathrm{H}_{2} \mathrm{O}$ & 0 & 0 & 200 & 410 \\
\hline $\mathrm{MgSO}_{4} .7 \mathrm{H}_{2} \mathrm{O}$ & 180 & 100 & 0 & 0 \\
\hline $\mathrm{NaCl}$ & 8000 & 5340 & 5800 & 5800 \\
\hline $\mathrm{NaHCO}_{3}$ & 225 & 270 & 0 & 42 \\
\hline $\mathrm{ZnSO}_{4} .7 \mathrm{H}_{2} \mathrm{O}$ & $34 \cdot 2$ & 0 & 0 & 0 \\
\hline \multicolumn{5}{|l|}{ Others } \\
\hline EDTA & 0 & 0 & 4 & 4 \\
\hline Oleate $\mathrm{Na}$ & $4 \cdot 8$ & 0 & 0 & 0 \\
\hline Polyvinyl alcohol & 0 & 600 & 20 & 5 \\
\hline Tween 40 & 0 & 16 & 0 & 0 \\
\hline Tween 80 & 0 & 0 & 20 & 25 \\
\hline Ägar & 10000 & 0 & 0 & 0 \\
\hline Agarose & 0 & 0 & 0 & 10000 \\
\hline
\end{tabular}

* Butler (1962); Wolin (1963); Herriott et al. (1970); GMT = complete medium (see Methods).

coagglutination test. One hundred and thirty-five $(29.1 \%)$ of the 464 strains that grew on GMT medium required histidine, $279(60 \cdot 1 \%)$ required hypoxanthine and 87 required both. Thirty-one strains $(6.7 \%)$ failed to grow on the aminoacid-thymidinedepleted medium. Of these, 15 required lysine, eight required methionine, seven required glycine, one required trytophan, and four required isoleucine or leucine in addition to one of the aminoacids listed above. Although some aminoacids stimulated growth of some of the isolates, problems of reproducibility made this effect valueless for typing purposes.

\section{Discrimination provided by chemotyping}

Table II shows the results of chemotyping for the 464 strains of $H$. influenzae that grew on GMT medium. A total of 56 chemotypes was recognised. The commonest types were $2,15,22,28,38$ and 52 , which contained respectively $20(4 \cdot 3 \%), 33(7 \cdot 1 \%)$, $66(14.6 \%), 40(8 \cdot 5 \%), 84(18 \cdot 1 \%)$ and $26(5 \cdot 6 \%)$ of the strains. Of the 27 strains that produced mucoid colonies, 24 (20 type $b$ and 4 of other capsular types) belonged to chemotype 15. Of the exceptions, one strain of type b (NCTC8467) required methionine and was assigned to chemotype 16, and two of other types belonged to chemotypes 10 and 28. Nine non-capsulated strains were assigned to chemotype 15 . Five of these non-capsulated strains were subcultured daily for 5 days on GMT medium, but none produced mucoid colonies. The type culture of $H$. influenzae (NCTC4560) belonged to chemotype 50. Of 256 strains tested, $18(7 \%$ ) produced $\beta$-lactamase; these 18 were distributed among 12 chemotypes. 
TABLE II

Distribution of chemotypes among 464 strains of H. influenzae

\begin{tabular}{|c|c|c|c|c|c|}
\hline Chemotype & Characters & $\begin{array}{c}\text { Number } \\
(\%) \text { of } \\
\text { strains }\end{array}$ & Chemotype & Characters & $\begin{array}{c}\text { Number } \\
(\%) \text { of } \\
\text { strains }\end{array}$ \\
\hline 1 & Zero* & $6(1 \cdot 3)$ & 29 & Hi-Hy-Gl-Ld & $1(0 \cdot 2)$ \\
\hline 2 & $\mathrm{Hi}$ & $20(4 \cdot 3)$ & 30 & Hi-Hy-Ly-Ld & $2(0 \cdot 4)$ \\
\hline 3 & Hy & $9(1.9)$ & 31 & Hi-Id-Od & $1(0 \cdot 2)$ \\
\hline 4 & Id & $2(0 \cdot 4)$ & 32 & Hi-Ur-Ld & $2(0.4)$ \\
\hline 5 & Od & $3(0 \cdot 6)$ & 33 & Hi-Od-Ld & $8(1 \cdot 7)$ \\
\hline 6 & Ld & $6(1 \cdot 3)$ & 34 & Hy-Id-Od & $3(0.6)$ \\
\hline 7 & $\mathrm{Hi}-\mathrm{Hy}$ & $16(3.4)$ & 35 & Hy-Ly-Id-Od & $1(0 \cdot 2)$ \\
\hline 8 & Hi-Hy-Ly & $3(0.6)$ & 36 & Hy-Id-Ld & $2(0 \cdot 4)$ \\
\hline 9 & $\mathrm{Hi}-\mathrm{Od}$ & $2(0.4)$ & 37 & Hy-Ur-Ld & $7(1 \cdot 5)$ \\
\hline 10 & $\mathrm{Hi}-\mathrm{Ld}$ & $14(3 \cdot 0)$ & 38 & Hy-Od-Ld & \\
\hline 11 & Hy-Ur & $6(1 \cdot 3)$ & 39 & Hy-Me-Od-Ld & $1(0 \cdot 2)$ \\
\hline 12 & Hy-Me-Is-Ur & $2(0.4)$ & 40 & Hy-Ly-Od-Ld & $1(0 \cdot 2)$ \\
\hline 13 & $\mathrm{Hy}-\mathrm{Od}$ & $19(4.0)$ & 41 & Id-Ur-Ld & $3(0 \cdot 6)$ \\
\hline 14 & Hy-Ly-Od & $2(0 \cdot 4)$ & 42 & Id-Od-Ld & $18(3.9)$ \\
\hline 15 & Hy-Ld & $33(7 \cdot 1)$ & 43 & Hi-Hy-Id-Ur & $1(0 \cdot 2)$ \\
\hline 16 & Hy-Me-Ld & $2(0 \cdot 4)$ & 44 & Hi-Hy-Id-Od & $2(0 \cdot 4)$ \\
\hline 17 & Hy-Gl-Is-Ld & $1(0 \cdot 2)$ & 45 & Hi-Hy-Me-Id-Od & $1(0 \cdot 2)$ \\
\hline 18 & Id-Od & $2(0 \cdot 4)$ & 46 & Hi-Hy-Id-Ld & $3(0 \cdot 7)$ \\
\hline 19 & Id-Ld & $1(0 \cdot 2)$ & 47 & Hi-Hy-Ly-Id-Ld & $1(0 \cdot 2)$ \\
\hline 20 & $\mathrm{Ur}-\mathrm{Ld}$ & $8(1 \cdot 7)$ & 48 & Hi-Hy-Ur-Ld & $3(0 \cdot 7)$ \\
\hline 21 & Me-Is-Ur-Ld & $1(0 \cdot 2)$ & 49 & Hi-Hy-Od-Ld & $1(0 \cdot 2)$ \\
\hline 22 & Od-Ld & $66(14 \cdot 6)$ & 50 & Hi-Id-Od-Ld & $3(0 \cdot 6)$ \\
\hline 23 & Gl-Od-Ld & $3(0 \cdot 6)$ & 51 & Hy-Id-Ur-Ld & $2(0 \cdot 4)$ \\
\hline 24 & Hi-Hy-Id & $1(0 \cdot 2)$ & 52 & Hy-Id-Od-Ld & $26(5 \cdot 6)$ \\
\hline 25 & Hi-Hy-Gl-Id & $2(0 \cdot 4)$ & 53 & Hy-Ly-Id-Od-Ld & $2(0 \cdot 4)$ \\
\hline 26 & Hi-Hy-Od & $2(0 \cdot 4)$ & 54 & Hy-Tr-Id-Ud-Ld & $1(0 \cdot 2)$ \\
\hline 27 & $\mathrm{Hi}-\mathrm{Hy}-\mathrm{Me}-\mathrm{Od}$ & $1(0 \cdot 2)$ & 55 & Hi-Hy-Id-Od-Ld & $8(1 \cdot 7)$ \\
\hline 28 & Hi-Hy-Ld & $40(8 \cdot 5)$ & 56 & Hi-Hy-Ly-Id-Od-Ld & $3(0 \cdot 6)$ \\
\hline
\end{tabular}

Typing characters used: Requirement for histidine (Hi), hypoxanthine (Hy), lysine (Ly), methionine (Me), glycine (Gl), tryptophan (Tr), isoleucine or leucine (Is); Negative result in tests for indole (Id), urease (Ur), ornithine decarboxylase (Od), lysine decarboxylase (Ld).

* None of the listed characters.

\section{Reliability of chemotyping}

In this laboratory, each sputum specimen is homogenised so that the sample cultured is representative of all parts of the specimen, and is plated before and after 5000-fold dilution (Tebbutt and Coleman, 1978). In this study, five colonies of $H$. influenzae from each of 14 purulent specimens of sputum were typed. In each case the five colonies from the same specimen were of the same chemotype. Twenty strains from various sources were retyped after being subcultured several times and being stored in liquid nitrogen, and the tests were carried out on several batches of the defined medium. No changes of type were noted.

\section{Relationship of chemotype to site of isolation}

All five strains isolated from the cerebrospinal fluids of infants with acute meningitis were of capsular type $b$, and were assigned to chemotype 15 . Three isolates were obtained from blood cultures. One of these, from a child with epiglottitis, was of capsular type $b$ and belonged to chemotype 15 . The other two were non-capsulated 
and of types 28 and 38; both were isolated from adults with respiratory disease, one of whom had acquired agammaglobulinaemia.

Six strains of $H$. influenzae were isolated from vaginal cultures. Three of these strains (belonging to types 38, 41 and 51) were obtained from children with vaginal discharge, two (type 46 and a strain that failed to grow on GMT medium) were associated with post-natal infections, and one (type 46) was isolated (together with Neisseria gonorrhoeae) from a patient with pelvic inflammatory disease.

Fig. 1 shows the common chemotypes of $H$. influenzae isolated from specimens of sputum (305 strains, including 39 from one ward—see below), from eyes ( 79 strains),

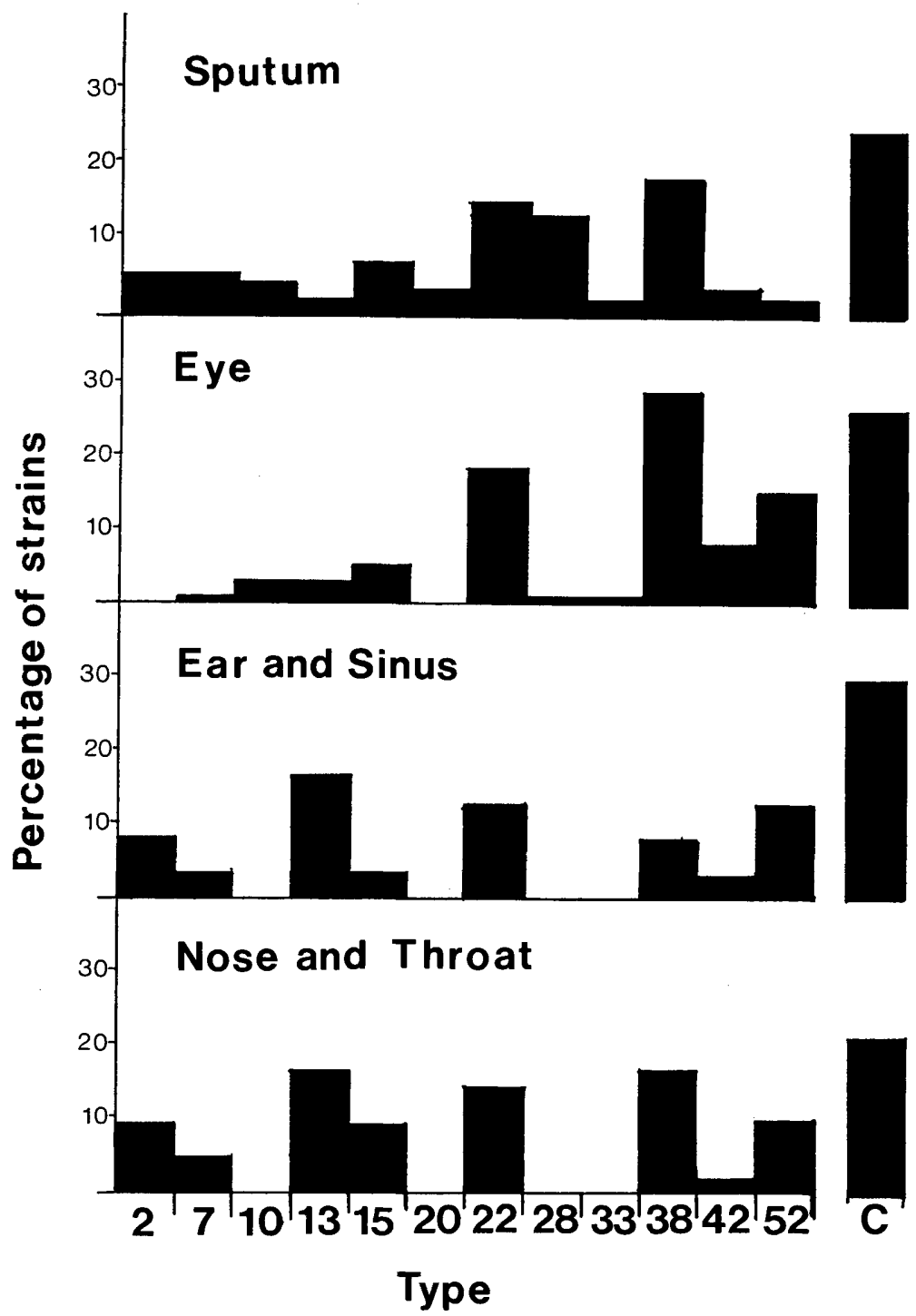

Fig. 1.-Frequency distribution of common chemotypes of $H$. influenzae according to the site of isolation. $\mathrm{C}=$ combined total of other chemotypes. 
from ears and sinuses ( 25 strains), and from noses and throats (41 strains). Strains belonging to type 22 and to type 38 were isolated frequently from all sites. Thirty-nine $(12.8 \%)$ of the 305 strains from sputum were of type 28 , but there was only one other isolate of this type (from an eye). Type 42 and type 52 strains accounted for $7 \cdot 6 \%$ and $13.9 \%$ of isolates from eye swabs respectively, but for only $3.3 \%$ and $2.3 \%$ of isolates from sputum specimens; $16.4 \%$ of strains from upper respiratory tract sites (ear, sinus, nose and throat), but only $1.6 \%$ of the isolates from sputum and $3 \%$ of those from eyes were of type 13. Thirteen strains of $H$. influenzae, of 12 different types, were isolated from nine patients with cystic fibrosis; nine $(69 \%)$ of them produced lysine decarboxylase, whereas only $23 \%$ of other respiratory-tract isolates did so.

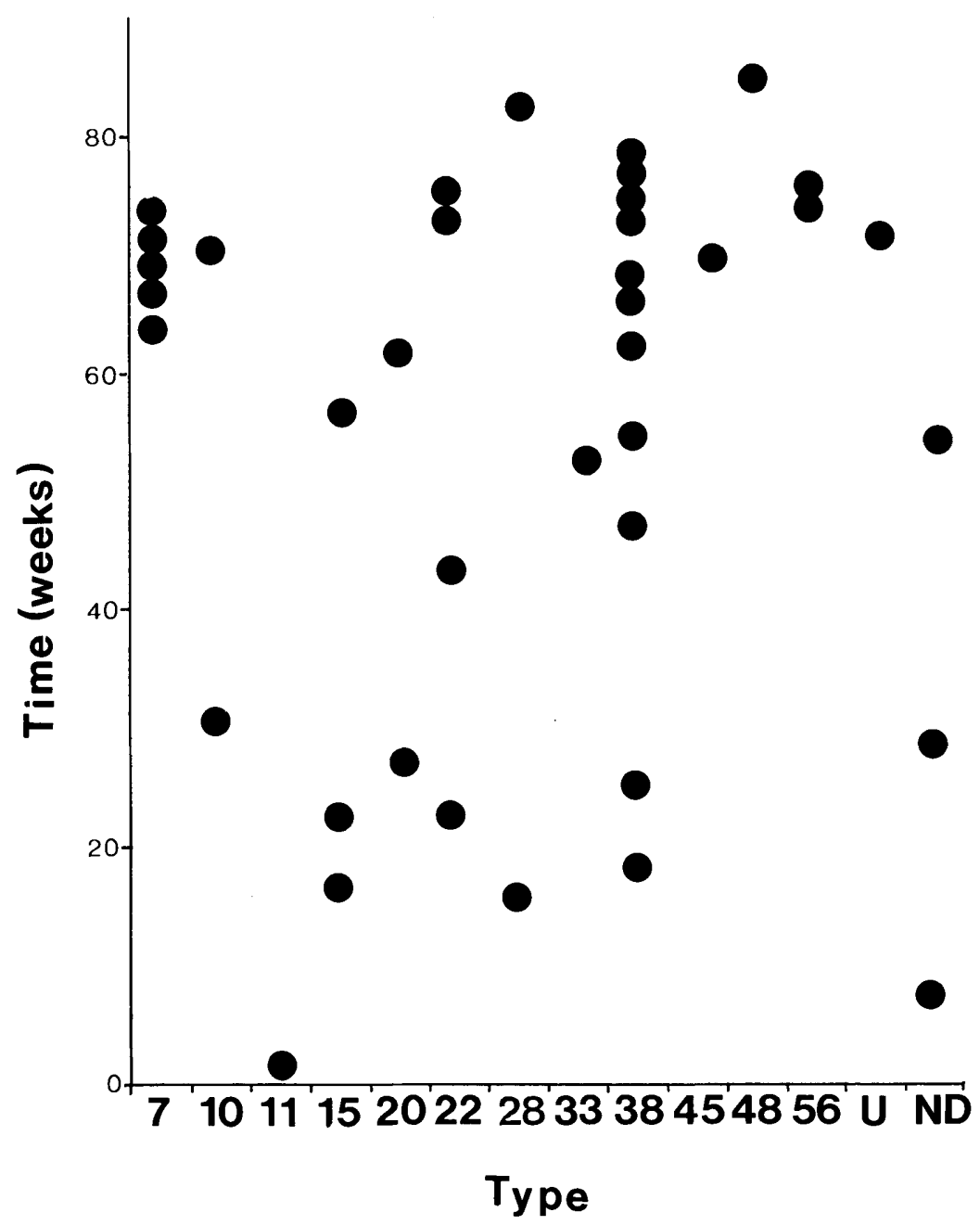

FIG. 2.-Frequency distribution of the chemotypes of $39 \mathrm{H}$. influenzae isolates from specimens of sputum from patients in a general surgical ward during a 20 -month period. $U=$ untypable; $N D=$ chemotype not determined. 


\section{Usefulness of chemotyping: selected clinical cases}

During a 20-month period, strains of $H$. influenzae isolated from the sputum of patients in a general surgical ward were studied. Of the 39 strains isolated, 35 were assigned to 12 chemotypes, one failed to grow on the defined medium, and three were not typed. Fig. 2 shows the type-distribution of these isolates. Typing results for the first 15 months showed little of interest, but after that there was an increase in the number of isolations of certain types. A corresponding increase in the number of post-operative chest infections was also reported. The isolation of $H$. influenzae of chemotype 7 from five patients suggests cross-infection, especially as they were the only type-7 strains isolated. All five patients smoked cigarettes, but none was a chronic bronchitic. All were assessed clinically as having post-operative chest infections requiring antimicrobial treatment and physiotherapy. $H$. influenzae type 56 was isolated from sputum specimens from two other patients with post-operative chest infections. Because only three strains of this type were isolated during the whole survey, cross-infection again seems probable. H. influenzae type 38 was isolated from the sputum of 11 patients on the surgical ward. Although there was an increase in the number of isolations of this type in the last few weeks of the survey, such strains were frequently isolated from other sputum specimens, and further tests may be needed to subdivide this type for epidemiological purposes.

\section{Discussion}

For epidemiological purposes, a typing system should be easily performed, reliable, reproducible, applicable to a large proportion of isolates and able to provide a useful degree of strain discrimination. The combination of tests described here was relatively easy to perform, interpret and reproduce. More than $97 \%$ of clinical isolates were typable. Although the discrimination achieved was considerably greater than that provided by biotyping alone or by the use of antibiograms, further subdivision of some of the common chemotypes would probably be an advantage.

There is little point in typing isolates unless they have been shown to be representative of the members of their species present in the specimen. This applies particularly to isolates from sputum, which may be contaminated with bacteria from the upper respiratory tract. In this study homogenisation helped to ensure that a representative sample of sputum was examined, and when tests were performed on several colonies from primary cultures of purulent specimens of sputum the results confirmed the presence of a single chemotype.

There was some correlation between chemotype and the site of isolation, but in most cases there were not enough strains in each group for this to be assessed reliably. Nearly all isolates from meningitis and bacteraemic illnesses belonged to chemotype 15 , but this is probably due to the concentration of capsular type b strains in this chemotype. It was observed that five of six isolates from the vagina did not produce indole whereas $81 \%$ of other strains did so. The chemotypes of genital isolates, although not necessarily identical, were, with one exception, very uncommon among strains from other sites. This finding suggests that genital strains are different from those that colonise the upper respiratory tract, as reported by Wallace et al. (1981). There is no evidence that the haemophili isolated here played a part in causing these 
vaginal infections, but a further study of their incidence, chemotype distribution and clinical importance may be worthwhile.

Laszlo et al. (1973) reported that up to $80 \%$ of patients after upper abdominal surgery suffer from chest complications. In such patients a profuse growth of $H$. influenzae, Streptococcus pneumoniae, or both is almost always associated with clinical evidence of respiratory infection (Wilkinson et al., 1977). These infections are probably caused by micro-organisms which the patient carries in the upper respiratory tract, but the results described here suggest that some are due to cross-infection. In patients with pre-existing chronic bronchial disease or a history of heavy smoking, infection is probably induced by anaesthesia or some other factor related to the operation. In cases of cross-infection, which usually develop later in the post-operative period, other determining factors must be important.

Another area in which chemotyping might provide clinically relevant information is in the study of $H$. influenzae strains associated with acute purulent exacerbations of chronic bronchitis. Chemotyping might make it possible to determine whether these strains are merely those commonly found in human upper respiratory tracts or are a special group, and whether recurrence of such purulent exacerbations is due to the persistence of the same strains or to fresh exogenous infections.

I thank Dr E. McKay-Ferguson for advice during the preparation of the manuscript. I am grateful to $\mathrm{Mr} \mathrm{W}$. M. Cooke and to Mr D. Clarke for access to the medical records of patients under their care. I also thank staff of the Control of Infection Department, South Tees Health Authority, in particular Mrs J. Reynolds and Mrs M. P. Johnson.

\section{REFERENCES}

Barenkamp S J, Munson R S, Granoff D M 1982 Outer membrane protein and biotype analysis of pathogenic nontypable Haemophilus influenzae. Infection and Immunity 36:535-540.

Butler L O 1962 A defined medium for Haemophilus influenzae and Haemophilus parainfluenzae. Journal of General Microbiology 27:51-60.

Clarke P H, Cowan S T 1952 Biochemical methods for bacteriology. Journal of General Microbiology 6:187-197.

Ellis D A, Anderson I M E, Stewart S M, Calder J, Crofton J W 1978 Exacerbations of chronic bronchitis: exogenous or endogenous infection. British Journal of Diseases of the Chest 72:115-121.

Glode M P, Daum R S, Goldmann D A, Leclair J, Smith A 1980 Haemophilus influenzae type B meningitis: a contagious disease of children. British Medical Journal 280:899-901.

Herriott R M, Meyer E Y, Vogt M, Modan M 1970 Defined medium for growth of Haemophilus influenzae. Journal of Bacteriology 101:513-516.

Kilian M 1974 A rapid method for the differentiation of Haemophilus strains. The porphyrin test. Acta Pathologica et Microbiologica Scandinavica 82B:835-842.

Kilian M 1976 A taxonomic study of the genus Haemophilus, with the proposal of a new species. Journal of General Microbiology 93:9-62.

Lautrop H 1960 Laboratory diagnosis of whooping cough or Bordetella infections. Bulletin of the World Health Organization 23:15-31.

Laszlo G, Archer G G, Darrell J H, Dawson J M, Fletcher C M 1973 The diagnosis and prophylaxis of pulmonary complications of surgical operation. British Journal of Surgery 60:129-134.

Malmvall B, Branefors-Helander P 1978 R-factor involvement in a local outbreak of ampicillin-resistant Haemophilus influenzae infections. Scandinavian Journal of Infectious Diseases 10:53-56.

Mehtar S, Law C A 1980 An outbreak of $\beta$ lactamase producing Haemophilus influenzae (biotype III) on a geriatric ward. Journal of Hospital Infection 1:357-358. 
Møller V 1955 Simplified tests for some amino acid decarboxylases and for the arginine dihydrolase system. Acta Pathologica et Microbiologica Scandinavica 36:158-172.

Tebbutt G M, Coleman D J 1978 Evaluation of some methods for the laboratory examination of sputum. Journal of Clinical Pathology 31:724-729.

Wallace R J, Musher D M, Septimus E J, McGowan J E, Quinones F J, Wiss K, Vance P H, Trier P A 1981 Haemophilus influenzae infections in adults: characterization of strains by serotypes, biotypes and $\beta$ lactamase production. Journal of Infectious Diseases 144:101-106.

Wilkinson P J, Ball A J, Doran J, Gillespie W A, Orton V S 1977 Routine laboratory assessment of postoperative chest infection: a prospective study. Journal of Clinical Pathology 30:417-420.

Wolin H L 1963 Defined medium for Haemophilus influenzae type b. Journal of Bacteriology 85:253-254. 